\title{
Chronische Lungenerkrankungen im Kindesalter
}

Chronische Lungenerkrankungen zählen nicht zu den häufigen Problemen im Kindesalter, stellen aber aufgrund der vielfältigen Differenzialdiagnosen eine große Herausforderung dar. Von einer „chronischen Problematik" spricht man im Allgemeinen bei einer Symptomdauer von mehr als 8 Wochen. Bei der Abklärung ist es sinnvoll, bereits bestehende Protokolle (Chang AB, Oppenheimer JJ, Weinberger MM et al. Chest 2017; 151: 875 - 883) zu berücksichtigen. Neben den spezifischen Symptomen können das Manifestationsalter sowie das klinische Setting
(Alter/Region) hinweisgebend sein (Chang AB, Oppenheimer J], Weinberger MM et al. Chest 2017; 152: 607 -617).

Nahezu alle chronischen Lungenerkrankungen im Kindesalter weisen die Leitsymptome Husten und/oder Tachypnoe auf. Die Ursachen eines chronischen Hustens dürfen nicht pauschal vom Erwachsenen- auf das Kindesalter übertragen werden; die Altersgrenze stellt hier das 15. Lebensjahr dar. Bei der Diagnosefindung sollte in Anamnese und Status auf spezifische Hinweiszeichen („cough pointers/red flags“) geachtet wer- den ( $\triangleright$ Tab. 1). Im Vortrag wird beispielhaft auf einige Krankheitsentitäten und deren spezifische Bildgebung eingegangen.

Chronische Lungenerkankungen mit verminderter Sekretolyse können bereits im Kindesalter zur Ausbildung von Bronchiektasen führen. Neben der Mukoviszidose (CF) als bekannter Ursache, soll im Vortrag die Abklärung von Non-CF-Bronchiektasen erläutert werden (Kurzidim L, Schwarz C, Schönfeld N et al. Der Pneumologe 2014; doi:10.1007/s10405-0130734-z).

- Tab. 1 Spezifische Hinweiszeichen bei chronischem Husten im Kindesalter (Chang AB, Oppenheimer IJ, Weinberger MM et al. Chest 2017; 151: 875 883) 2019 UpToDate $^{\circledR}$ : Signs and symptoms suggesting a specific cause of cough in children (specific cough „pointers“).

\section{spezifische Hinweiszeichen}

\section{Anamnese}

1. pulmonale Symptome

produktiver Husten

Hämoptysen

Giemen/pfeifende Ausatmung

Tachypnoe/Dyspnoe

typischer Hustencharakte mögliche ursächliche Krankheit

suppurative Lungenerkrankung

Tuberkulose

Asthma bronchiale

interstitielle Lungenerkrankung, bronchopulmonale Dysplasie

Mucoid Impaction, Pertussis 


\section{- Tab. 1 (Fortsetzung)}

\section{spezifische Hinweiszeichen}

rezidivierende Pneumonien

2. Manifestationszeit und Trigger

Symptomatik seit der Neugeborenen-Periode

Symptomatik seit einem Aspirationsereignis

Husten ändert sich bei Ablenkung oder Angst/Anspannung

Exposition gegenüber Tbc oder Haustieren, Reiseanamnese

3. assoziierte Grunderkrankung

Herzfehler mit Links-Rechts-Shunt

Muskelerkrankungen Stoffwechselerkrankungen

gastroösophagealer Reflux

Sichelzellanämie

Mukoviszidose

Autoimmunerkrankung

körperlicher Status

Trommelschlegelfinger

Thorax-Deformität

Stridor, Giemen, Rasselgeräusche

auffällige Routineuntersuchung

Röntgen-Thorax

Lungenfunktion

Sauerstoffsättigung

\section{mögliche ursächliche Krankheit}

Immundefekt, Sekretolyse-Störung

bronchopulmonale Dysplasie

Fremdkörper-Aspiration

Vocal-Cord-Dysfunction, habitueller Husten

\section{Interessenkonflikt}

Die Autoren geben an, dass kein Interessenkonflikt besteht.
Autorinnen/Autoren

\section{Kurzidim}

Kinderklinik Westbrandenburg Potsdam

Korrespondenzadresse
Bibliografie

DOI https://doi.org/10.1055/a-0943-1213

Online-Publikation: 2019

Fortschr Röntgenstr 2019; 191: S115-S116

(c) Georg Thieme Verlag KG, Stuttgart · New York ISSN 1433-5972

\section{Kurzidim}

Kinderklinik Westbrandenburg Potsdam

ludwikkurzidim@gmx.net 